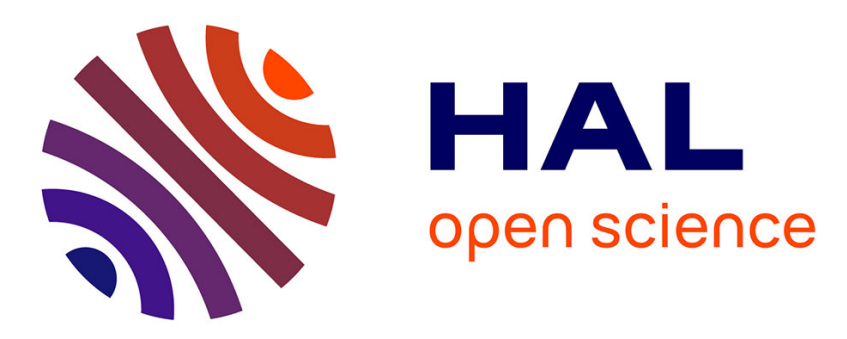

\title{
Une nouvelle station de mesure pour l'acquisition de séries chronologiques en hydrologie urbaine
}

N. Walcker, Jean-Luc Bertrand-Krajewski, S. Vacherie, M. Lepot, H. Castebrunet, Sylvie Barraud, G. Lipeme Kouyi

\section{- To cite this version:}

N. Walcker, Jean-Luc Bertrand-Krajewski, S. Vacherie, M. Lepot, H. Castebrunet, et al.. Une nouvelle station de mesure pour l'acquisition de séries chronologiques en hydrologie urbaine. Techniques Sciences Méthodes , 2018, 3, pp.55 - 64. 10.1051/tsm/201803055 . hal-01739774

\author{
HAL Id: hal-01739774 \\ https://hal.science/hal-01739774
}

Submitted on 22 Mar 2018

HAL is a multi-disciplinary open access archive for the deposit and dissemination of scientific research documents, whether they are published or not. The documents may come from teaching and research institutions in France or abroad, or from public or private research centers.
L'archive ouverte pluridisciplinaire $\mathbf{H A L}$, est destinée au dépôt et à la diffusion de documents scientifiques de niveau recherche, publiés ou non, émanant des établissements d'enseignement et de recherche français ou étrangers, des laboratoires publics ou privés. 


\title{
Une nouvelle station de mesure pour l'acquisition de séries chronologiques en hydrologie urbaine ${ }^{1}$
}

\author{
A new monitoring station for the acquisition of time series in urban hydrology \\ Walcker N. ${ }^{1,2 *}$, Bertrand-Krajewski J.-L. ${ }^{1}$, Vacherie S. ${ }^{1}$, Lepot M. ${ }^{3}$, \\ Castebrunet H. ${ }^{1}$, Barraud S. ${ }^{1}$, Lipeme Kouyi G. ${ }^{1}$
}

1. Université de Lyon, INSA Lyon, laboratoire DEEP, EA 7429, 34 avenue des Arts, F-69621 Villeurbanne cedex

2. GRAIE, Campus LyonTech La Doua, 66 bd Niels Bohr, CS 52132, F69603 Villeurbanne cedex

3. TU Delft, Civil Engineering and Geosciences, Water management and Sanitary Engineering, Stevinweg 1 (Building 23), 2628 CN Delft, The Netherlands

*nicolas.walcker@graie.org

Mots-clés : métrologie, acquisition de données, autosurveillance, pilotage temps réel

Keywords: metrology, data acquisition, monitoring, real-time control

\section{Introduction}

L'OTHU (Observatoire de Terrain en Hydrologie Urbaine - www.othu.org), créé en 1999, fédère douze équipes de recherche de neufs établissements, en collaboration étroite avec la Métropole de Lyon et l'agence de l'eau Rhône Méditerranée et Corse. Ces équipes exploitent quotidiennement des milliers de données obtenues à l'aide d'une instrumentation riche et diversifiée ayant pour vocation le suivi de la quantité et de la qualité des eaux urbaines sur de nombreux sites expérimentaux (réseaux unitaires, réseaux séparatifs, milieux aquatiques superficiels et souterrains, techniques de gestion des eaux pluviales à la source).

Trois sites de l'OTHU (voir Figure 1), à savoir une rivière périurbaine et un déversoir d'orage à Grézieula-Varenne, un déversoir d'orage à Ecully et un système de rétention-infiltration des eaux pluviales à Chassieu, possèdent des stations de mesure sur réseau d'assainissement équipées de capteurs assurant le suivi de la qualité des effluents (conductivité électrique, température, $\mathrm{pH}$, turbidité) placés dans un canal de dérivation alimenté en continu à l'aide d'une pompe (voir Figure 2).

\footnotetext{
${ }^{1}$ Paru dans TSM, 2018, 3, 55-64. doi 10.1051/tsm/201803055.
} 


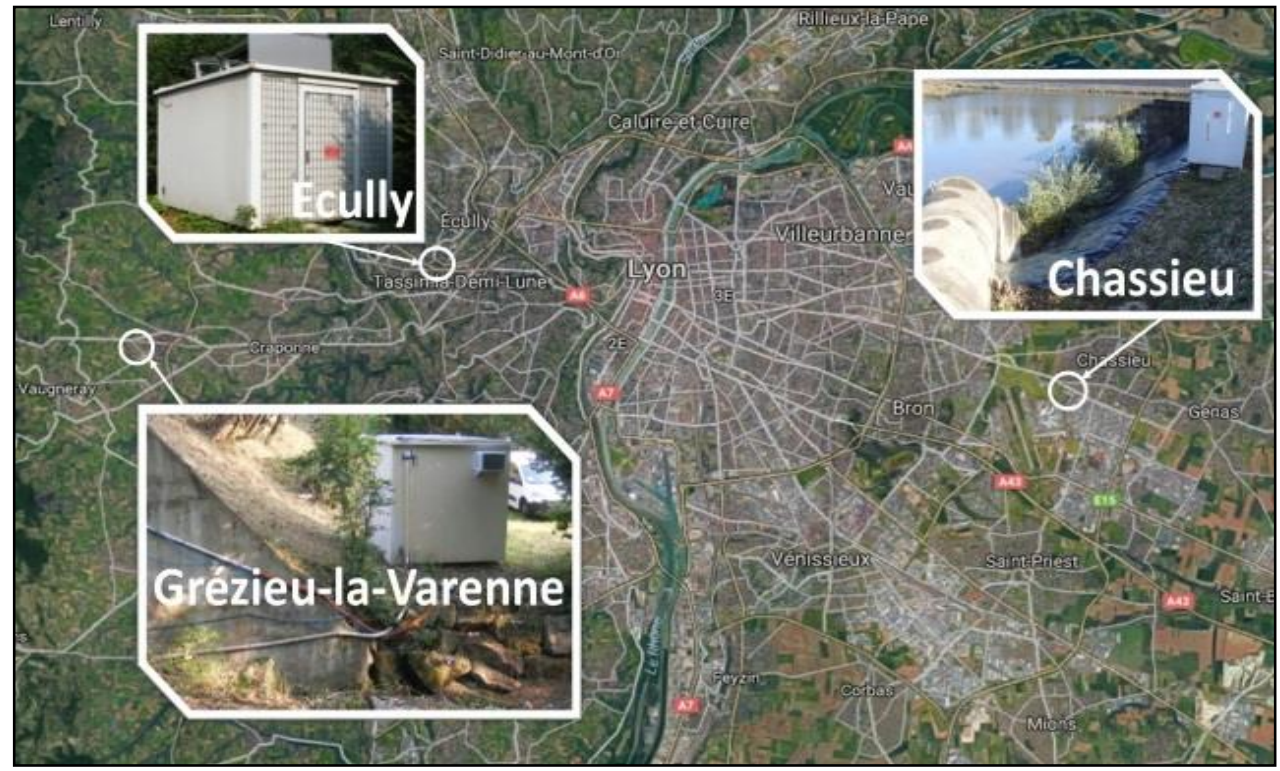

Figure 1. Localisation des sites OTHU sur réseau d'assainissement équipés pour le suivi en continu.

Des capteurs mesurant les hauteurs d'eau, vitesses d'écoulement et débits au sein des réseaux, ainsi que des pluviographes, complètent ce parc métrologique. Les données sont collectées par différentes centrales d'acquisition et transmises à l'aide de divers outils (GSM, ligne téléphonique, carte $\mathrm{SD}, \ldots$...

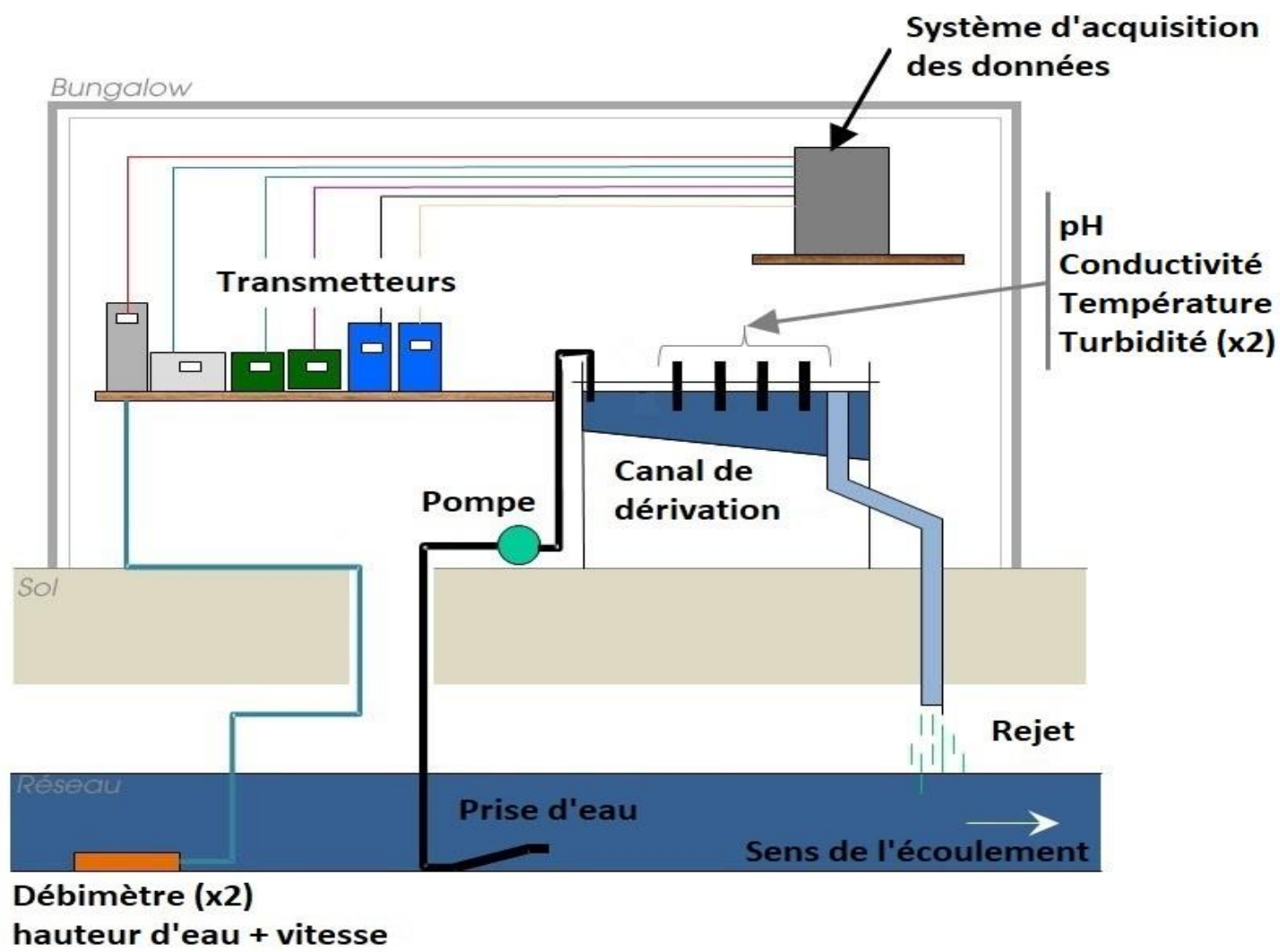

Figure 2. Schéma type d'une station de mesure OTHU (d'après Mourad, 1999).

Ces stations servent deux objectifs : 1) acquérir des données pour les projets de recherche en appui sur l'OTHU, et 2) fournir des données pour l'autosurveillance réglementaire de la Métropole de Lyon. Les plus anciennes ont été opérationnelles en 2002 et, après une dizaine d'années de fonctionnement, malgré 
des adaptations et évolutions indispensables, il était devenu nécessaire de les rénover entièrement pour les rendre plus adaptables aux nouveaux standards de communication et plus performantes (acquisition à courts pas de temps).

C'est dans ce cadre et dans une démarche d'amélioration continue que l'équipe OTHU du laboratoire DEEP (Déchets Eaux Environnement Pollutions) de l'Institut National des Sciences Appliquées de Lyon - INSA Lyon a apporté ces dernières années de nombreuses améliorations sur les stations de mesure. Les principaux objectifs étaient les suivants :

- améliorer la qualité des données brutes ;

- augmenter les possibilités d'acquisition de données et de pilotage des stations ;

- optimiser la maintenance des sites ;

- réduire les coûts de fonctionnement ;

- améliorer le transfert des résultats vers les opérationnels.

Dans cet article, nous présentons la nouvelle génération des stations OTHU et montrons le déploiement d'une station métrologique type sur l'un des sites majeurs de l'OTHU : le bassin de rétention Django Reinhardt à Chassieu. Les paragraphes suivants présentent successivement le site, le matériel utilisé, les différentes évolutions apportées à la station de mesure, les résultats obtenus et quelques perspectives.

\section{Matériels et méthodes}

\subsection{Dix ans d'expérience sur le site de Chassieu}

Le bassin versant de Chassieu aboutit à un bassin de rétention par une canalisation circulaire d'un diamètre de $1.60 \mathrm{~m}$, avec une pente de $1 \%$, dans laquelle se situe un ensemble de capteurs mesurant les hauteurs d'eau ( 3 capteurs de hauteur par ultrasons) et les vitesses d'écoulement ( 2 capteurs de vitesse Doppler et 1 capteur radar). A proximité immédiate de cette canalisation se situe la station de mesure (bungalow installé en surface, Figure 2) équipée de capteurs de qualité des eaux (pH, conductivité électrique, turbidité, température) placés au sein d'un canal de dérivation alimenté par une pompe.

L'intérêt de l'enregistrement de longues séries chronologiques est indéniable pour le suivi à court pas de temps de la qualité des eaux et pour estimer les charges polluantes (de Bénédittis et BertrandKrajewski, 2006 ; Métadier et Bertrand-Krajewski, 2010, 2012 ; Lombard et al., 2010 ; Lacour et al., 2010) ou proposer une gestion en temps réel basée sur la qualité des effluents (Lacour et al., 2009).

Malgré leurs caractéristiques spécifiques bien adaptées aux besoins, les premières stations OTHU (Mourad, 1999 ; Barraud et al., 2002), au cours des dernières années de leur exploitation, ont montré leurs limites : le stockage devenu insuffisant des données (en taille et formats), la consultation différée des données, l'identification laborieuse des maintenances, peu ou pas de possibilités de pilotage à distance, le défaut de représentativité des données en cas de nettoyage, panne ou déplacement d'un capteur, les données parfois bruitées par la présence de perturbations électromagnétiques (en particulier en raison de l'usage de la pompe d'alimentation fonctionnant sous $380 \mathrm{~V}$ ). Ces constats et les nombreuses évolutions technologiques récentes ont conduit l'OTHU à proposer une nouvelle génération de stations, dont la première a été mise en œuvre sur le site de Chassieu.

\subsection{Nouvelle conception fondée sur les retours d'expérience}

Fort d'une décennie d'utilisation quotidienne de la première génération de stations de mesure, de nombreuses améliorations, tant matérielles que méthodologiques, ont été apportées à la conception de la seconde génération. 


\subsubsection{Changements de matériels}

Le Tableau 1 présente les principales différences entre les deux générations de stations.

Par temps de pluie, du sable peut être transporté depuis le réseau jusqu'au bungalow et décanter au niveau des capteurs, perturbant ainsi les mesures. La nouvelle section ovoïde du canal de mesure optimise l'écoulement et réduit significativement la décantation des particules. Afin de limiter l'effet d'une possible sédimentation résiduelle de solides au niveau des capteurs, un système de nettoyage automatique est intégré (buse avec eau potable et vidange pilotable, Figure 3) dans le radier du canal (le séquençage du nettoyage est précisé dans la partie suivante).

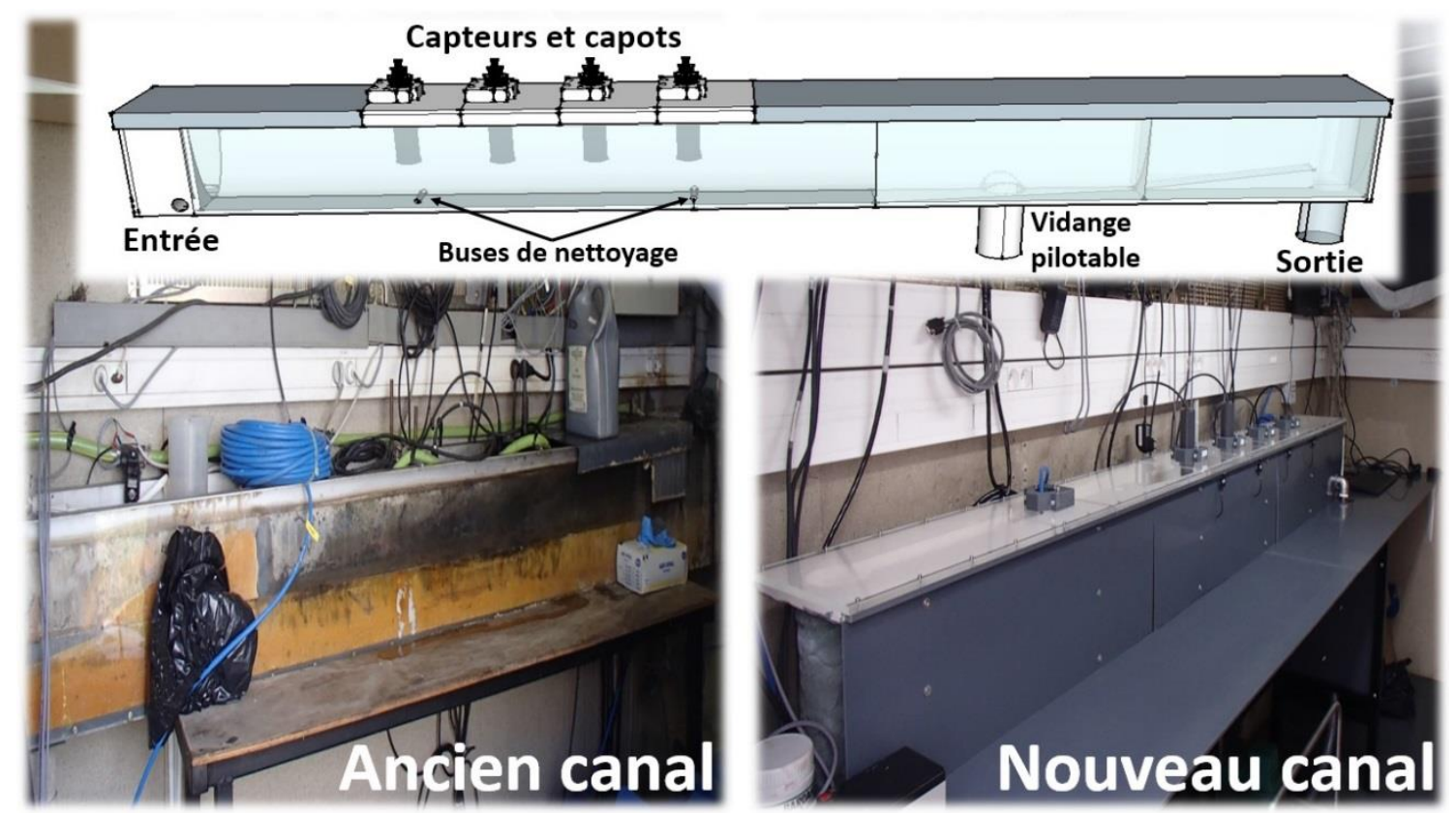

Figure 3. Conception du nouveau canal de dérivation et différences entre un ancien et un nouveau canal.

La commande de la pompe péristaltique d'alimentation de l'ancienne station en mode "pompage refoulement - arrêt » et à vitesse constante, quelles que soient les conditions hydrauliques dans le réseau, engendrait des à-coups et des phénomènes d'entrainement d'air, tous deux responsables d'usures prématurées des tubes de pompe et de dysfonctionnements hydrauliques du système. L'ajout d'un variateur de fréquence sur la nouvelle station limite l'usure de la pompe (cavitation, obstruction, échauffement) et des consommables (lobes, plaque d'usure, éléments mécaniques), réduit la fréquence des maintenances et les coûts associés.

La remise en place des sondes après chaque maintenance (nettoyage, étalonnage) était parfois non répétable avec l'ancien système utilisant une noix de laboratoire et une pince trois doigts : la hauteur et l'orientation des sondes par rapport à l'écoulement n'étaient pas toujours constantes. Pour résoudre ce problème, des capots transparents avec un système de fixation rapide et répétable des capteurs garantissent désormais leur bon positionnement par rapport au fond et aux parois du canal de dérivation (Figure 4). Ces capots améliorent également la reproductibilité des mesures en réduisant l'impact de remises en place différentes par les différents intervenants sur site. Les maintenances elles-mêmes, autrefois notées dans des cahiers de site (avec parfois des oublis, des erreurs, des mauvais horodatages...), sont maintenant détectées automatiquement : des contacts magnétiques contrôlent en continu le bon positionnement des capteurs avec une détection instantanée de tout changement d'état (ouverture et fermeture du capot, mauvaise remise en place des capteurs, maintenance en cours). 
Robustes (étanchéité et pas de partie mobile) et bon marché (moins de 10€), ces contacts sont une solution idéale pour remplacer les cahiers de site.

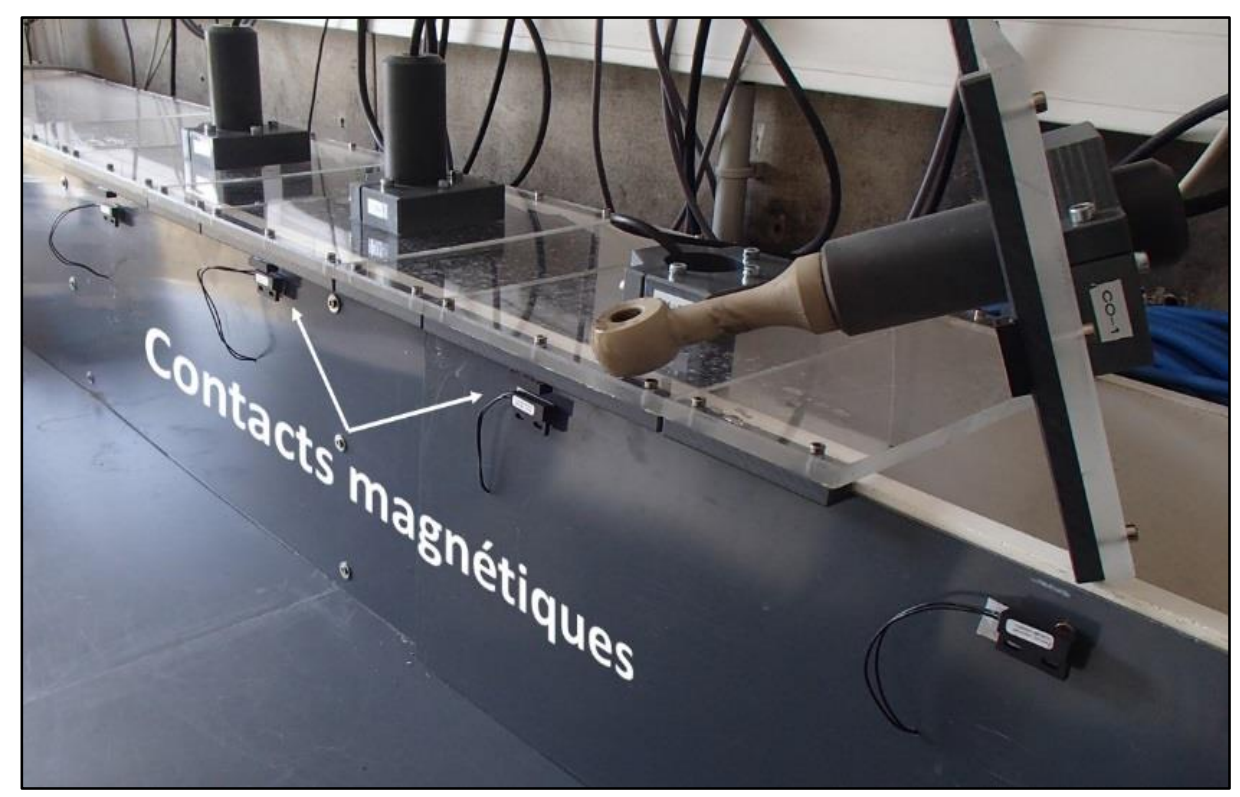

Figure 4. Canal de dérivation, capots et contacts magnétiques.

Le système d'acquisition de données, fondamental dans les stations, a été entièrement repensé. L'ancien système composé d'une centrale SOFREL était limité en termes de types d'entrées, de sorties, de fréquence d'acquisition et de possibilités de prétraitement des données. Il est dorénavant composé d'un ordinateur équipé de différentes cartes d'acquisition National Instruments, implémentées sur un châssis. D'abord testé en laboratoire, ce matériel permet l'acquisition et le pilotage d'un grand nombre de paramètres en parallèle à des cadences particulièrement élevées (liées aux performances des processeurs de l'ordinateur), une grande liberté dans le choix des entrées et des sorties grâce à la modularité du châssis Instruments et plus de flexibilité dans l'évolution de l'instrumentation. L'utilisation d'un ordinateur pour l'acquisition des données accroît notablement les possibilités par rapport aux centrales d'acquisition classiques : capacité de stockage accrue, prétraitement en temps réel des données, taille et format des données non limités. Avec des interfaces graphiques codées (Figure 5) sous Labview®, la station devient intégralement pilotable en temps réel en local et à distance via une connexion ADSL Assymetric Digital Subscriber Line et un VPN - Virtual Private Network, et utilisable par des utilisateurs non-initiés (la centrale SOFREL utilisait une interface texte de type MS-DOS). 


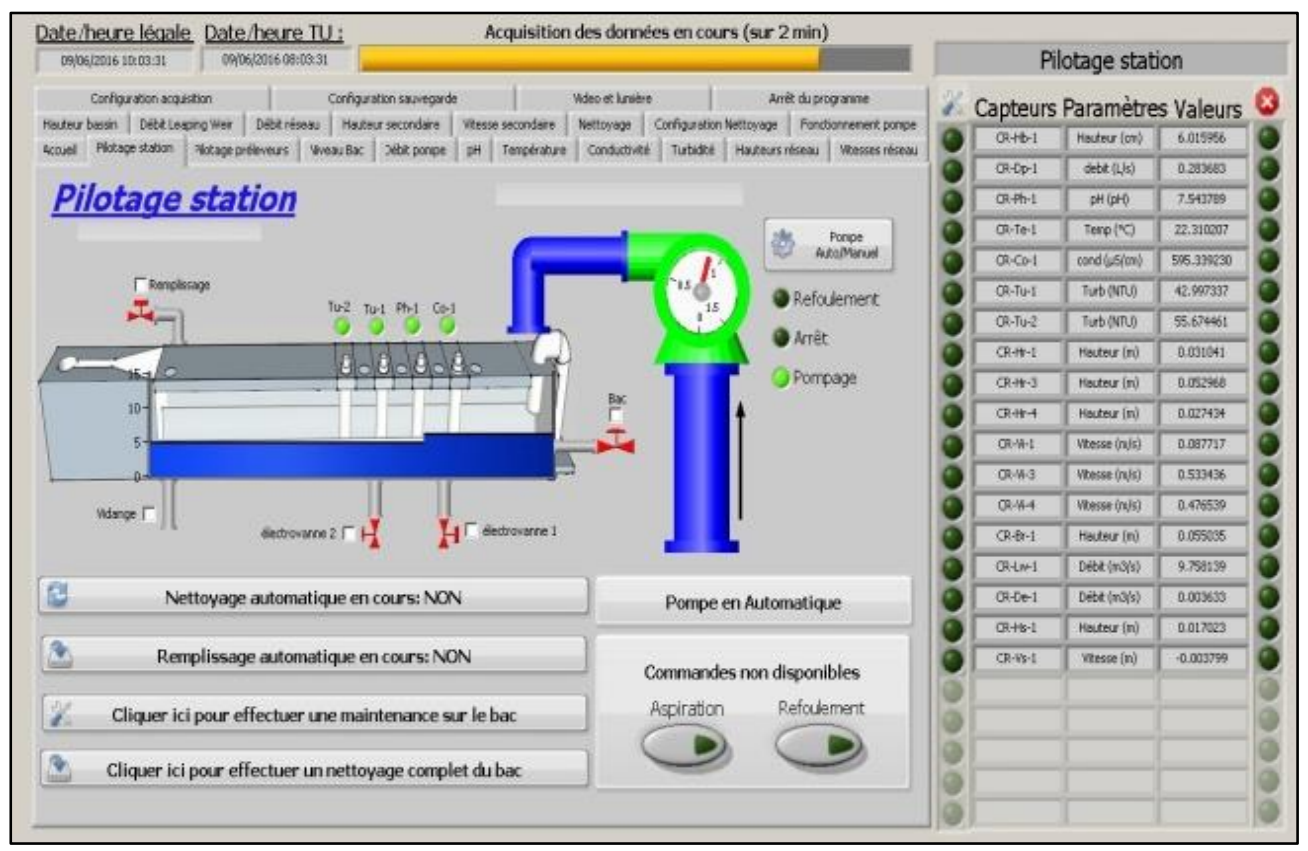

Figure 5. Extrait de l'Interface Homme Machine développée sous Labview ${ }^{\circledR}$

L'expérience et l'analyse de données de débit ont aussi montré le manque de fiabilité des données de débit, en particulier pour les faibles hauteurs d'eau inférieures à $7 \mathrm{~cm}$. Afin d'y remédier, un débitmètre électromagnétique a été installé sur un déversoir d'orage de type leaping-weir. Ce dispositif (Figure 6) permet de mesurer précisément les faibles débits jusqu'à $4 \mathrm{~L} / \mathrm{s}$ dans le collecteur d'arrivée.

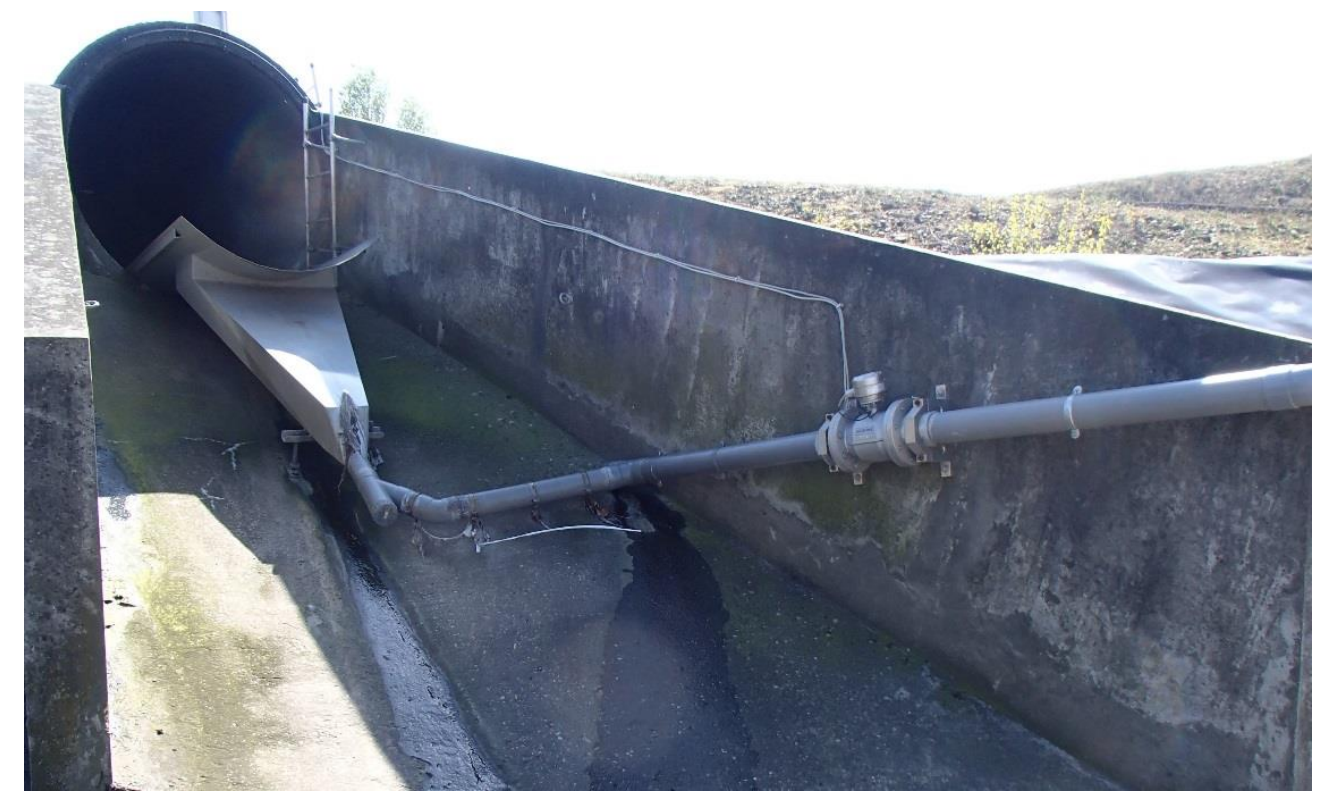

Figure 6. Dispositif pour le mesurage des faibles débits (leaping weir et débitmètre électromagnétique).

D'autres améliorations plus minimes ont été mises en place : climatisation réversible (température mieux contrôlée pour les conditions de travail, d'étalonnage et la durabilité des appareils), nouvelle pompe à lobes (moins de maintenance et de dysfonctionnements dus aux tubes), installation d'une webcam pour un pré-diagnostic à distance.

\subsubsection{Adaptation des méthodes}

Le changement de matériels implique des changements de méthodes. Le nouveau système autorise des acquisitions d'entrées analogiques jusqu'à $200 \mathrm{kHz}$ (limité à $1 \mathrm{~Hz}$ ici) et permet un prétraitement des 
données avant leur enregistrement. L'ancienne station enregistrait une valeur ponctuelle prise toutes les deux minutes par capteur. Un nouvel algorithme de prétraitement des données au pas de 2 minutes est mis en place. Les valeurs sont acquises au pas de la seconde pendant 2 minutes, puis leur médiane est calculée. Ensuite, $70 \%$ des valeurs initiales centrées autour de la médiane sont conservées et leur moyenne est calculée. Ce retrait de $30 \%$ des valeurs acquises au pas de la seconde permet d'éliminer les valeurs extrêmes, anormales et/ou aberrantes, notamment celles qui correspondent au temps nécessaire (environ $35 \mathrm{~s}$ ) au nettoyage d'une sonde, tout en assurant une moyenne très fiable au pas de 2 minutes car calculée avec plus de 80 valeurs. Cet algorithme limite considérablement l'impact des erreurs aléatoires et permet d'obtenir une donnée brute prétraitée au pas de 2 minutes davantage représentative de la qualité de l'effluent mesurée sur cette période.

L'ensemble des actions et évènements sur la station est pris en compte dans le contrôle de la représentativité de l'échantillon (maintenance des capteurs, panne éventuelle de la pompe, nettoyage, ...). L'interface graphique permet le prétraitement des données en identifiant automatiquement et instantanément les valeurs non représentatives de la qualité de l'effluent (pompe en panne, nettoyage en cours, etc.) arrivant dans le bassin de rétention. Cela permet un gain de temps important dans la validation des données (passage de $25 \%$ à $15 \%$ d'un équivalent temps plein) et prévient des erreurs d'interprétation.

Le pilotage à distance et en temps réel à l'aide de l'interface homme machine (Figure 5) permet meilleure réactivité des utilisateurs et limite les interventions nécessaires sur site (ex: possibilité d'activer un nettoyage du canal à distance). La connexion ADSL et le VPN facilitent la consultation du fonctionnement de la station, à tout moment, en tout lieu et en temps réel.

Toutes ces méthodes sont exécutées en parallèle, en temps réel et de façon entièrement autonome. La station contrôle en continu ses propres conditions de fonctionnement (démarrage de la pompe sur évènements pluvieux, nettoyage automatique par temps sec, détection des maintenances curatives nécessaires et des pannes, etc.) et informe instantanément les opérateurs par courriels des anomalies rencontrées. On limite ainsi considérablement l'impact d'une panne de communication ou l'absence d'un superviseur tout en optimisant le nombre d'interventions sur site.

\section{Résultats et discussion}

\subsection{Vers une efficacité accrue des outils opérationnels}

Les trois voies d'amélioration proposées ont permis de réduire considérablement le temps de main d'œuvre nécessaire à la maintenance.

Les capacités de télé-opération et télé-présence réduisent le nombre de déplacements sur site, la mobilisation du personnel et donc les coûts de fonctionnement de la station. Une courte visite de contrôle hebdomadaire d'une trentaine de minutes remplace les précédentes interventions de maintenance (nettoyage bac et capteurs, détection pannes...) réalisées tous les deux jours par une équipe technique composée de deux personnes. Cette configuration offre également une meilleure réactivité limitant ainsi le fonctionnement en mode dégradé de la station et donc la quantité de données potentiellement manquantes en cas de dysfonctionnement prolongé.

Après un an de fonctionnement, le changement de type de pompe (pompe à lobes en replacement d'une pompe péristaltique) et de son mode de commande (variation de fréquence et inversion de phase) ont 
permis de n'avoir plus aucune maintenance à réaliser sur la partie hydraulique. A titre de comparaison, la précédente installation nécessitait un changement bimensuel du tube de la pompe péristaltique.

Le canal de dérivation, le système de nettoyage et les capots assurent de meilleures conditions de mesure (répétabilité et reproductibilité) et de fonctionnement des capteurs, tout en facilitant les interventions sur le site et en améliorant l'hygiène et la sécurité pour les intervenants. L'automatisation a permis de diviser par 6 la fréquence de nettoyage réduisant ainsi la consommation d'eau courante, l'usure du matériel (les électrovannes sont moins sollicitées) et les biais dans les données dus aux dilutions par l'eau de lavage du canal.

\subsection{Des données brutes de meilleure qualité}

La différence entre les deux méthodes d'acquisition et de traitement des données est illustrée Figure 7, qui montre la comparaison entre un signal instantané acquis au pas de 2 minutes avec l'ancienne station OTHU et un signal obtenu avec le nouvel algorithme et la nouvelle station. Il apparaît clairement un gain dans la qualité des données, plus intégratives sur 2 minutes et avec un signal moins bruité.

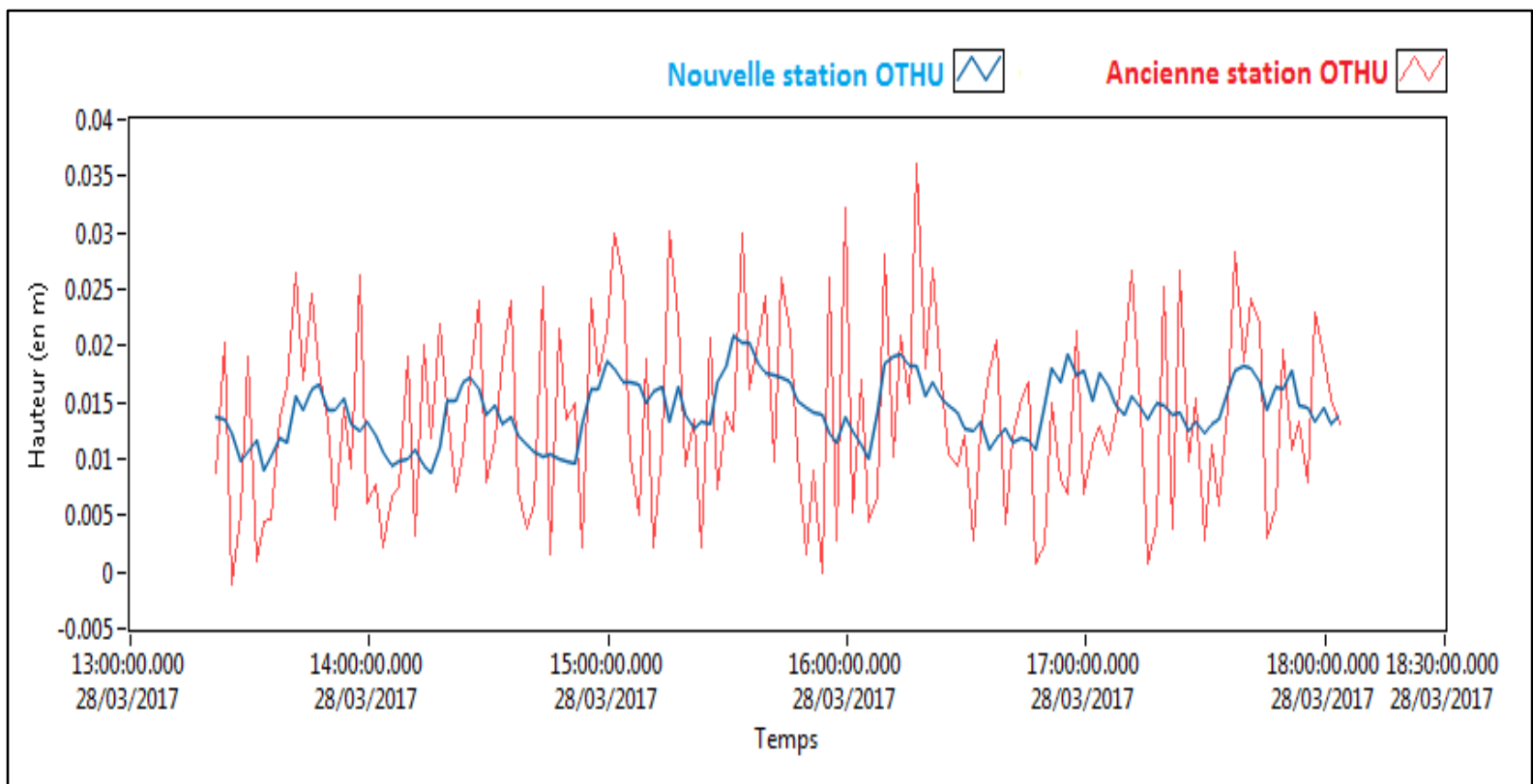

Figure 7. Comparaison de données au pas de 2 minutes : en rouge, acquisition d'une valeur instantanée au pas de 2 minutes (ancienne station OTHU) ; en bleu, valeur de la moyenne sur 2 minutes à partir de $70 \%$ des valeurs acquises au pas de la seconde et centrées autour de la médiane (nouvelle station OTHU).

Concernant les mesures de débit, les capteurs en réseau (hauteur d'eau par ultrasons et vitesse d'écoulement par radar) ont été maintenus aux mêmes emplacements qu'avec l'ancienne station. Ils sont fixés sur la voûte du collecteur de $1.6 \mathrm{~m}$ de diamètre. Le prétraitement des données de débit a permis l'identification de cycles de débits de temps sec d'une périodicité de 30 min environ (Figure 8), indétectables par l'ancienne station (Figure 9) qui délivrait des signaux trop bruités pour l'identification de ces cycles. 


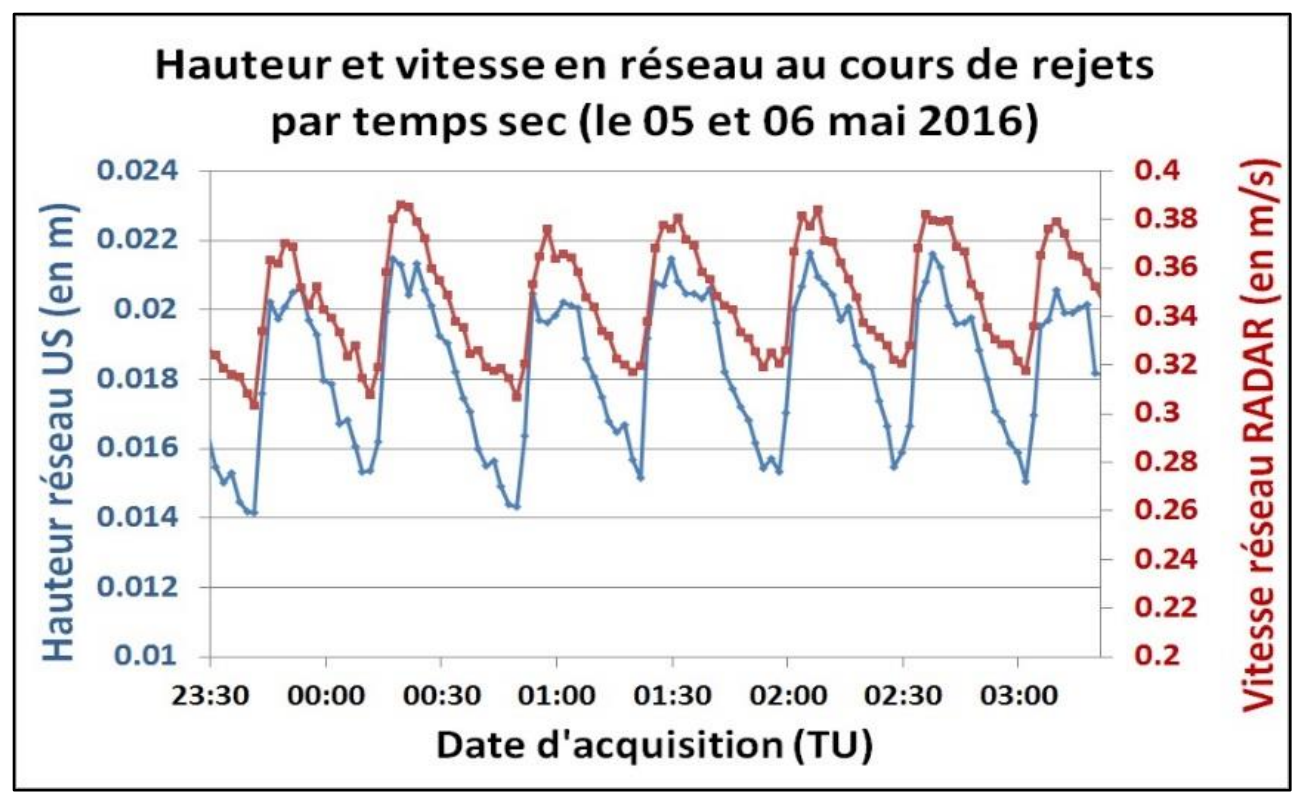

Figure 8. Hauteur d'eau et vitesse d'écoulement par temps sec avec la nouvelle station.

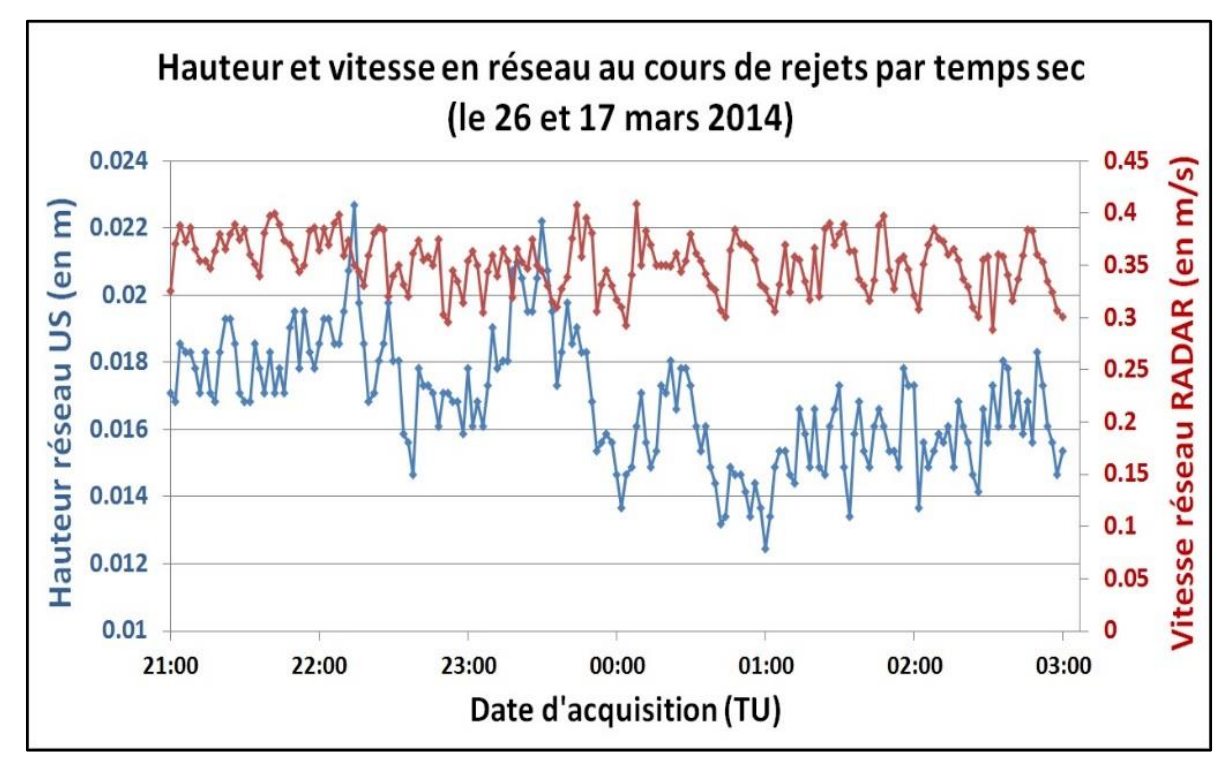

Figure 9. Hauteur d'eau et vitesse d'écoulement par temps sec avec l'ancienne station.

Ces cycles sont confirmés par les mesures du débitmètre électromagnétique sur le leaping-weir (Figure 10), indiquant la présence de ce cycle d'environ 30 minutes observé par les capteurs de hauteur et vitesse. 


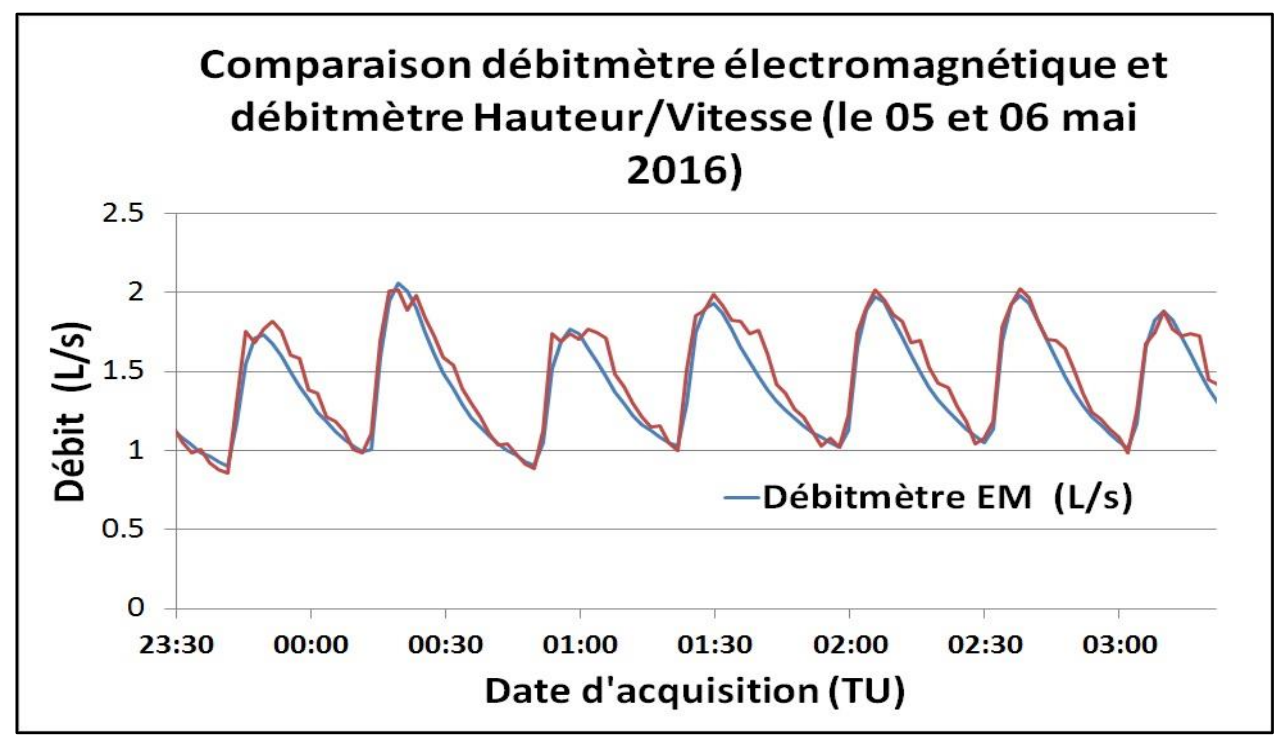

Figure 10. Comparaison des débits mesurés de temps sec (électromagnétique vs. hauteur/vitesse)

Ces résultats nous apportent de nouveaux éléments de compréhension du fonctionnement hydraulique du site de Chassieu et d'interprétation des données relatives à la qualité de l'effluent au cours des évènements pluvieux. En effet, l'impact du débit de temps sec est observable sur la qualité de l'échantillon mesuré par temps de pluie. La Figure 11 illustre les données du pH (en bleu) et de conductivité électrique (en rouge) obtenues au cours de l'évènement pluvieux de la soirée du 5 avril 2016. 


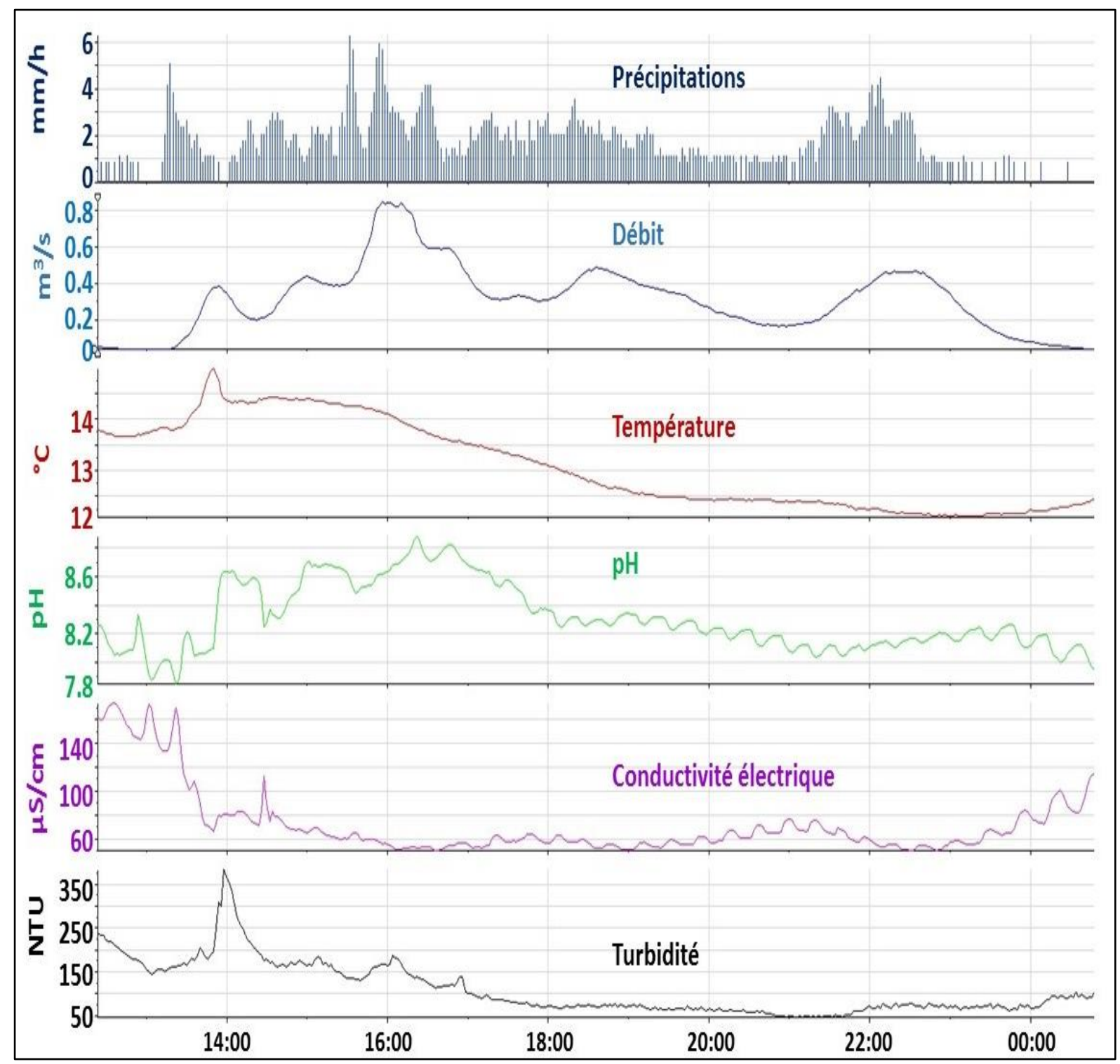

Figure 11. Suivi des paramètres quantitatifs et qualitatifs au cours de l'évènement pluvieux du 5 avril 2016 (pas de temps d'enregistrement : 2 minutes)

Un phénomène cyclique dans les chroniques de $\mathrm{pH}$ et de conductivité électrique est clairement visible au cours de la seconde partie de cet évènement, à partir de 18h00. Ce cycle témoigne de l'impact perceptible des rejets continus par temps sec (Figure 10) sur les paramètres de qualité malgré un débit moyen d'approximativement $300 \mathrm{~L} / \mathrm{s}$. Ce phénomène ne semble pas observable sur les chroniques de température et de turbidité. Il est également intéressant de noter les faibles variations mesurées des paramètres physico-chimiques au cours de chaque cycle (moins de $10 \mu \mathrm{S} / \mathrm{cm}$ pour la conductivité électrique et moins de 0.1 unité pour le $\mathrm{pH}$ ).

\subsection{Discussion}

Le choix du matériel d'acquisition, l'usage d'un ordinateur, de cartes d'acquisition et de logiciels de programmation nous permettent de nous affranchir des matériels ou logiciels commerciaux parfois trop bridés et limités dans leur compatibilité. L'absence de connectiques propriétaires spécifiques à certaines marques augmente également les possibilités d'instrumentation. 
La qualité des données brutes a été grandement améliorée sans changer de capteurs, de transmetteurs, d'emplacements ou d'installation mais simplement en modifiant notre méthode d'acquisition et de prétraitement des données. Cela souligne l'importance d'une maitrise globale de la chaine d'acquisition, de la mise en place du capteur jusqu'à la valeur finale enregistrée. En améliorant la représentativité des données et en réduisant le bruit des signaux associés, de faibles variations et fluctuations deviennent observables et permettent de mieux comprendre le fonctionnement du bassin versant et des ouvrages et d'avoir de nouvelles données sur les phénomènes qui s'y déroulent. Cependant, cette démarche n'est pas suffisante pour garantir la production de données de qualité. Il est avant tout nécessaire de savoir sélectionner du matériel adapté aux besoins, de l'installer correctement sur un emplacement opportun et d'être en mesure de le vérifier et de l'étalonner régulièrement (Bertrand-Krajewski, 2013). Dans la situation présentée dans cet article, la capacité du matériel choisi répond à des fins de recherche et pourrait paraître surdimensionnée pour certaines applications opérationnelles (fréquence d'échantillonnage élevée, vitesse d'exécution du programme). Dans tout projet d'acquisition de données, il reste donc primordial de définir les objectifs visés afin de définir les besoins métrologiques et méthodologiques adaptés.

Enfin, trop souvent sous-estimée, l'intervention humaine reste indispensable pour garantir le bon fonctionnement des installations. Les conditions particulières et difficiles en hydrologie urbaine requièrent un suivi régulier et rigoureux. D'une façon générale, la métrologie nécessite une critique permanente des données produites, en particulier dans un environnement extérieur non contrôlé. La mise en place de la démarche présentée ici nécessite également l'évolution des compétences des utilisateurs en matière d'informatique, de programmation, de métrologie et d'automatisation.

\section{Conclusion et perspectives}

La nouvelle génération de stations de mesure de l'OTHU mise en place à Chassieu en 2015 permet une double amélioration de son exploitation opérationnelle et de la qualité des données acquises. Les conditions de travail au sein de la station de mesure ont été optimisées (propreté du site, interventions réduites, facilitées et ciblées). Le pilotage en temps réel améliore la réactivité et les opportunités d'actions. Le traitement des urgences et aléas s'oriente vers davantage de maintenance préventive et planifiée. Les faibles débits sont mieux mesurés et diffèrent seulement de $5 \%$ par rapport à ceux obtenus par un débitmètre électromagnétique. La qualité accrue des données améliore notre compréhension du fonctionnement du bassin versant. Le prétraitement des données en temps réel (détection des maintenances, détection des valeurs anormales et calcul des moyennes, etc.) simplifie le travail de validation ultérieur. Les erreurs aléatoires sont considérablement atténuées et les valeurs non représentatives rapidement identifiées.

Les outils informatiques utilisés, plus performants que les centrales d'acquisition classiques, offrent de nombreuses possibilités, notamment en termes d'automatisation, d'autonomie du site, de prétraitement avancé des données ou de pilotage d'un réseau de sites en temps réel. Les données acquises sont diversifiées : aux valeurs classiques des capteurs peuvent s'ajouter des signaux d'autocontrôle, des images, des sons, des vidéos ainsi que des données produites par tous les systèmes pouvant interagir entre eux et contrôlables par des ordinateurs. A l'avenir de nouveaux outils tels que l'hydrométrie vidéo pourraient se généraliser et compléter les futurs parcs métrologiques en hydrologie urbaine.

Les avancées technologiques actuelles offrent également des objets toujours plus connectés et autonomes. Les données produites, devenant de plus en plus nombreuses et variées, pourraient trouver dans le Big Data un moyen d'exploitation intéressant. 


\section{Remerciements}

Le travail présenté dans cet article a été effectué dans le cadre de l'Observatoire de Terrain en Hydrologie Urbaine (OTHU), site urbain de la ZABR (Zone Atelier Bassin de Rhône), avec le soutien de ses équipes membres, de la Métropole de Lyon, de l'Agence de l'eau Rhône Méditerranée Corse et de l'ensemble de ses partenaires. Nous remercions plus particulièrement Dominique Babaud et Serge Naltchayan (laboratoire DEEP, INSA Lyon) pour leur contribution à l'installation, l'évolution et la maintenance des stations de mesure OTHU, Laëtitia Bacot (GRAIE) pour la coordination administrative et la gestion concertée des interventions sur les sites OTHU, Patrick Lucchinacci et Christian Surgot (Direction de l’Eau de Lyon Métropole) pour leur disponibilité.

\section{Références bibliographiques}

Barraud S., Gibert J., Winiarski T., Bertrand-Krajewski J.-L. (2002). Implementation of a monitoring system to measure impact of stormwater runoff infiltration. Water Science and Technology, 45(3), 203210.

Bertrand-Krajewski J.-L. (2013). Auto-surveillance des réseaux d'assainissement : fiabiliser la métrologie, les données et leur exploitation. L'eau, l'industrie, les nuisances, 362, 84-89.

de Bénédittis J., Bertrand-Krajewski J.-L. (2006). Mesurage des concentrations en MES et DCO dans les eaux usées par spectrométrie UV/visible. La Houille Blanche, 4, 136-142.

Lacour C., Chebbo G., Joannis C. (2010). Évaluation de flux de polluants dans un réseau unitaire à partir de mesures en continu de turbidité. TSM, 1-2, 47-53.

Lacour C., Joannis C., Chebbo G. (2009). Amélioration potentielle de ta gestion des effluents de temps de pluie grâce à des mesures de turbidité. TSM, 7-8, 50-58.

Lombard V., Toloméo S., Bertrand-Krajewski J.-L., Debray R., Comte C., de Bénédittis J. (2010). Conception et mise en place de stations de mesure des flux polluants dédiées à la gestion intégrée d'un système d'assainissement. Actes de Novatech 2010, Lyon, France, 27 juin - 1 juillet, 10 p.

Métadier M., Bertrand-Krajewski J.-L. (2010). Traitement de séries chronologiques de turbidité continues à court pas de temps pour l'estimation des masses de MES et de DCO rejetées en milieu urbain par temps de pluie. La Houille Blanche, 2, 77-85.

Métadier M., Bertrand-Krajewski J.-L. (2012). The use of long-term on-line turbidity measurements for the calculation of urban stormwater pollutant concentrations, loads, pollutographs and intra-event fluxes. Water Research, 46(20), 6836-6856.

Mourad M. (1999). OTHU: Etalonnage des appareils de mesure et premier regard sur le traitement, la critique et la validation de données. Villeurbanne (France) : INSA Lyon, Rapport URGC-HU, 264 p. 


\title{
Résumé
}

La généralisation de la métrologie et de l'autosurveillance réglementaire en hydrologie urbaine ainsi que les conditions d'exploitation souvent difficiles nécessitent des outils toujours plus performants. C'est pourquoi l'OTHU (Observatoire de Terrain en Hydrologie Urbaine) a souhaité réhabiliter ses stations de mesure. Cet article illustre quelques innovations technologiques et méthodologiques mises en place afin d'assurer une production de données continues fiables tout en réduisant la maintenance nécessaire au bon fonctionnement de ces installations. La technologie actuelle offre de réelles opportunités en termes de réduction des coûts de maintenance, de réactivité, de prétraitement des données. Chaque station produit en moyenne 12240 données par jour afin d'assurer le suivi en continu de la quantité et de la qualité des eaux pluviales en milieu urbain. Le suivi de la dynamique des phénomènes sur le long terme nécessite du matériel disponible et opérationnel $24 \mathrm{~h} / 24$ et $7 \mathrm{j} / 7$. Les nouvelles stations OTHU sont fiables et fournissent des données représentatives et de meilleure qualité que les anciennes installations.

\begin{abstract}
The deployment of smart metrological procedures and practices as well as challenging operating conditions for urban water monitoring require ever more efficient tools. In this context, the OTHU (Field Observatory for Urban Water Management) rehabilitated its field monitoring stations. This paper aims at demonstrating benefits from innovative OTHU's monitoring stations regarding the reliability of collected data and the decrease of maintenance costs. The current technology offers real opportunities in terms of reduced costs, reactivity and improvement of data pre-processing. Each station produces approximately 12240 data per day, ensuring continuous monitoring of stormwater quantity and quality in urban environment. The long term monitoring of the dynamics of hydrological processes requires 24/7 operational and reliable equipment and devices. The new OTHU monitoring stations are reliable and provide representative data of higher quality than the previous ones
\end{abstract}




\begin{tabular}{|c|c|c|}
\hline & 1ère génération & 2ème génération \\
\hline Canal de dérivation & Section circulaire & Section ovoïde \\
\hline $\begin{array}{l}\text { Nettoyage automatique } \\
\text { du canal }\end{array}$ & Non & Oui, par buses et vanne de vidange \\
\hline $\begin{array}{c}\text { Remplissage automatique } \\
\text { d'eau dans le canal }\end{array}$ & Oui, à pas de temps fixe & Oui, en fonction des niveaux d'eau \\
\hline $\begin{array}{l}\text { Système de vidange du } \\
\text { canal }\end{array}$ & Non & Oui à l'aide d'une vanne pilotable \\
\hline $\begin{array}{l}\text { Détection des } \\
\text { maintenances }\end{array}$ & Non & $\begin{array}{c}\text { Oui, par contacteurs magnétiques et } \\
\text { programme Labview }\end{array}$ \\
\hline $\begin{array}{c}\text { Positionnement répétable } \\
\text { des capteurs }\end{array}$ & Non & Oui, par capots \\
\hline $\begin{array}{c}\text { Alimentation en eau } \\
\text { du canal }\end{array}$ & Pompe péristaltique & Pompe à lobes \\
\hline $\begin{array}{l}\text { Pilotage de la pompe en } \\
\text { fonction des conditions } \\
\text { dans le réseau }\end{array}$ & Non & Oui \\
\hline $\begin{array}{l}\text { Pilotage du débit } \\
\text { de la pompe }\end{array}$ & Non & Oui, avec variateur de fréquence \\
\hline Système d'acquisition & Centrale d'acquisition & PC, châssis et cartes d'acquisition \\
\hline $\begin{array}{l}\text { Communication } \\
\text { avec la station }\end{array}$ & Ligne téléphonique RTC & Ligne ADSL \\
\hline $\begin{array}{l}\text { Pilotage distant } \\
\text { en temps réel }\end{array}$ & Non & Oui \\
\hline $\begin{array}{c}\text { Pas de temps de } \\
\text { scrutation et d'acquisition }\end{array}$ & 2 minutes et 2 minutes & 1 seconde et 2 minutes \\
\hline Acquisition vidéo & Non & Oui, avec webcam ou caméra \\
\hline Sauvegarde des données & $\begin{array}{c}\text { Mémoire locale limitée à } \\
\text { quelques jours }\end{array}$ & $\begin{array}{l}\text { Mémoire locale importante et } \\
\text { extensible (sauvegarde } \\
\text { complémentaire sur le Cloud) }\end{array}$ \\
\hline
\end{tabular}

Tableau 1. Principales différences matérielles entre les deux générations de station 